\title{
Ultrasonido endoscópico (USE) y colangio- pancreatografía endoscópica retrógrada (CPRE) sin fluoroscopia en el tratamiento de la coledocolitiasis durante el embarazo: reporte de 2 casos
}

\section{Endoscopic ultrasound (EUS) and endoscopic retrograde cholangiopancreatography (ERCP) without fluoroscopy for the treatment of choledocholithiasis during pregnancy: Report of 2 cases}

Rómulo Darío Vargas-Rubio, ${ }^{1}$ (] Óscar Mariano Pinto-Saavedra. ${ }^{{ }^{*}}$ []

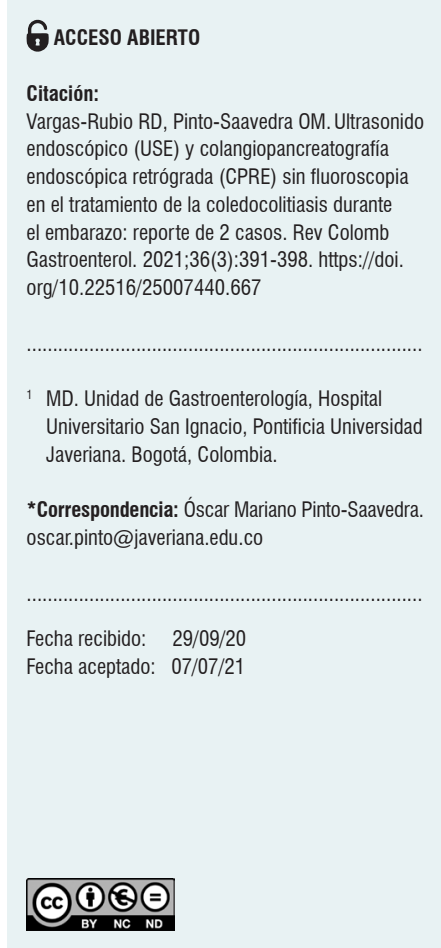

\section{Resumen}

La enfermedad litiásica biliar es una patología frecuente en mujeres embarazadas, y las complicaciones relacionadas con los cálculos biliares durante el embarazo pueden generar desenlaces adversos tanto en la madre como en el feto. La coledocolitiasis en el embarazo requiere de una aproximación diagnóstica adecuada y su manejo busca minimizar los riesgos de las intervenciones médicas. Se describen dos casos de mujeres embarazadas quienes presentan coledocolitiasis documentada por colangiorresonancia. Se realizó el tratamiento con la combinación de ultrasonido endoscópico (USE) y colangiopancreatografía endoscópica retrógrada (CPRE) sin fluoroscopia, con lo cual se logró resolver la coledocolitiasis sin exponer al feto a radiación ionizante, se confirmó la permeabilización del colédoco y se observó una adecuada evolución posoperatoria tanto materna como fetal.

\section{Palabras clave}

Coledocolitiasis, embarazo, colangiopancreatografía endoscópica retrógrada, endosonografía.

\begin{abstract}
Biliary lithiasis is a common condition in pregnant women, and complications related to gallstones during pregnancy can lead to adverse outcomes in both the mother and the fetus. Choledocholithiasis during pregnancy requires an adequate diagnostic approach to minimize the risks of medical interventions. The following are two cases of pregnant women with choledocholithiasis diagnosed using magnetic resonance cholangiography. Treatment included a combination of endoscopic ultrasound and retrograde endoscopic cholangiopancreatography (ERCP) without fluoroscopy, achieving the resolution of choledocholithiasis, without exposing the fetus to ionizing radiation, confirming the permeabilization of the common bile duct, and observing an adequate postoperative evolution of both the mother and the fetus.
\end{abstract}

Keywords

Choledocholithiasis; Pregnancy; Retrograde Endoscopic Cholangiopancreatography (ERCP); Endosonography.

\section{INTRODUCCIÓN}

La enfermedad litiásica biliar es más frecuente en las mujeres embarazadas pues está precipitada por el alto influjo hormonal (aumento de estrógenos y progesterona), que favorece la disminución en la motilidad de la vesícula biliar y el aumento del colesterol saturado en la bilis, fenómenos fisiopatológicos que facilitan la formación de cálculos y sus complicaciones asociadas como la coledocolitiasis ${ }^{(1)}$. La enfermedad litiásica biliar en el embarazo se asocia 
con parto pretérmino y morbilidad neonatal y materna, así como el aumento en la readmisión materna y neona$\operatorname{tal}^{(2)}$. Se estima una prevalencia de la enfermedad litiásica vesicular durante el embarazo entre $3,3 \%$ y $12 \%{ }^{(3)}$. Las complicaciones relacionadas con los cálculos biliares son frecuentes durante el embarazo, llegando a ser la segunda indicación más común para la intervención quirúrgica no obstétrica. Se estima una incidencia de barro biliar y colelitiasis entre $5 \%$ a $31 \%$ y $2 \%$ a $11 \%$, respectivamente ${ }^{(4)}$. Las complicaciones de la enfermedad litiásica vesicular durante el embarazo, entre las que se incluyen colecistitis, pancreatitis aguda y colangitis, se presentan en alrededor de $10 \%$; siendo la incidencia estimada de la coledocolitiasis poco frecuente, de 1/1000 embarazos, y se logra la resolución de la coledocolitiasis con colangiopancreatografía endoscópica retrógrada (CPRE) en el $100 \%$ de los casos en las series revisadas ${ }^{(3-5)}$.

Estos reportes de casos se realizaron siguiendo la estrategia CARE (CAse REport) ${ }^{(6)}$, la cual pretende ilustrar cómo en la actualidad disponemos de técnicas de intervención de la vía biliar seguras y efectivas en el tratamiento de la coledocolitiasis en mujeres embarazadas, sin la exposición a la radiación propia de la fluoroscopia.

\section{CASO 1}

Paciente de 31 años con antecedente de preeclampsia leve, quien cursa con un embarazo de 27 semanas, ingresa por un cuadro clínico de 4 días de evolución caracterizado por dolor epigástrico tipo ardor, asociado con dolor opresivo en región dorsal y lumbar. Concomitantemente, tiene múltiples episodios eméticos de contenido alimentario e intolerancia a la vía oral. $\mathrm{Al}$ examen físico presenta: presión arterial (PA): 113/69 mm Hg; frecuencia cardíaca (FC): 87 latidos por minuto $(\mathrm{lpm})$; frecuencia respiratoria (FR): 18 respiraciones por minuto (rpm); temperatura $(\mathrm{T}): 36,2$ ${ }^{\circ} \mathrm{C}$; saturación de oxígeno $\left(\mathrm{SatO}_{2}\right): 95 \%$; peso: $92 \mathrm{~kg}$. Conjuntivas rosadas, escleras ictéricas, útero grávido no doloroso, sin signos de irritación peritoneal, extremidades sin edemas, sin déficit neurológico. Se toman paraclínicos de ingreso, en los que se documenta hiperbilirrubinemia con patrón colestásico (Tabla 1).

Se realiza una colangiorresonacia (Figura 1) que documenta coledocolitiasis con efecto obstructivo y dilatación de la vía biliar intra- y extrahepática, y colelitiasis con signos de colecistitis. Se considera que la paciente embarazada presenta colelitiasis más colecistitis y coledocolitiasis, por lo que se decide llevar a un ultrasonido endoscópico (USE)-CPRE. Se realiza USE, el cual evidencia una segunda porción del duodeno de aspecto usual, colédoco con calibre de $6 \mathrm{~mm}$ de diámetro, en la porción media una imagen hiperecogénica que proyecta una sombra acústica posterior compatible con un cálculo de $5 \mathrm{~mm} \times 9 \mathrm{~mm}$ de diámetro y vesícula distendida con múltiples defectos que generan una sombra acústica posterior. En este sentido, el resultado del estudio es compatible con coledocolitiasis y colelitiasis (Figura 2). Por último, se retira el USE radial y se inserta un duodenoscopio.

Tabla 1. Paraclínicos de ingreso para el caso 1

\begin{tabular}{|lccc|}
\hline \multicolumn{1}{c}{ Examen } & Resultado & Unidades & \multicolumn{2}{c|}{ Valor de referencia } \\
\hline TGO-AST & 41 & $\mathrm{U} / \mathrm{L}$ & $0-35$ \\
\hline TGP-ALT & 44 & $\mathrm{U} / \mathrm{L}$ & $0-35$ \\
\hline Bilirrubina total & 3,97 & $\mathrm{mg} / \mathrm{dL}$ & $0,3-1$ \\
\hline Bilirrubina directa & 2,73 & $\mathrm{mg} / \mathrm{dL}$ & $0,0-0,2$ \\
\hline Bilirrubina indirecta & 1,24 & $\mathrm{mg} / \mathrm{dL}$ & $0,0-1,1$ \\
\hline Creatinina & 0,46 & $\mathrm{mg} / \mathrm{dL}$ & $0,5-1,0$ \\
\hline Amilasa & 86 & $\mathrm{U} / \mathrm{L}$ & $22-80$ \\
\hline Fosfatasa alcalina & 215 & $\mathrm{U} / \mathrm{L}$ & $30-120$ \\
\hline
\end{tabular}

ALT (TGP): alanina-aminotransferasa; AST (TGO): aspartatoaminotransferasa.

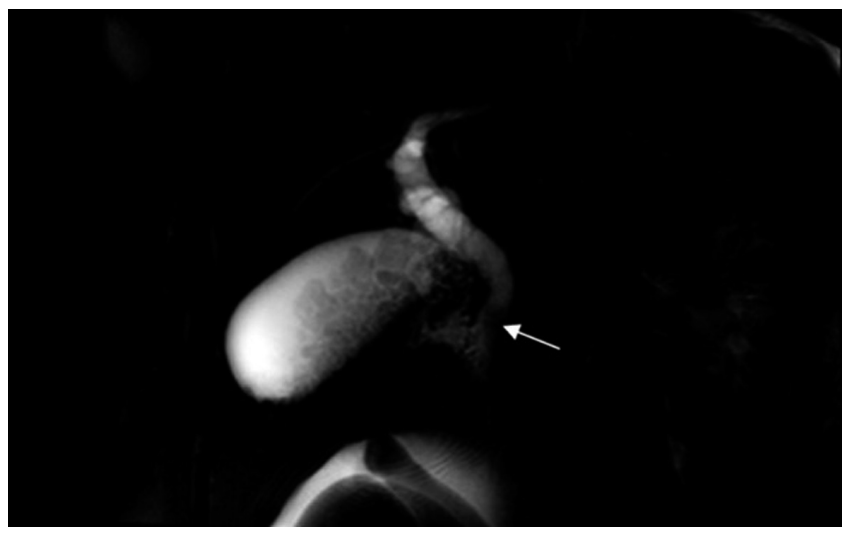

Figura 1. Colangiorresonancia. A nivel de la ampolla de Vater, se observa un defecto de $6 \times 4 \mathrm{~mm}$ (flecha) en su luz compatible con coledocolitiasis. Imagen propiedad de los autores.

Se aplican criterios de Tokio para la colangitis:

- El criterio A: hace referencia a la presencia de inflamación sistémica definida por fiebre $>38^{\circ} \mathrm{C}$, escalofrío o conteo de leucocitos $<4000$ o $>10000^{(7)}$; criterio no cumplido en este caso.

- El criterio B: se define como la presencia de ictericia con bilirrubina igual o mayor a 2 o la alteración de las pruebas de función hepática (transaminasas, fosfatasa alcalina y gamma-glutamil transferasa elevadas más de 1,5 veces el límite superior de la normalidad; criterio que sí se cumple en este caso ${ }^{(7)}$. 
- El criterio C: describe la dilatación de la vía biliar por imagen o presencia de causa obstructiva en las imágenes (cálculos, estenosis, stent) ${ }^{(7)}$; este criterio sí se cumple en este caso.

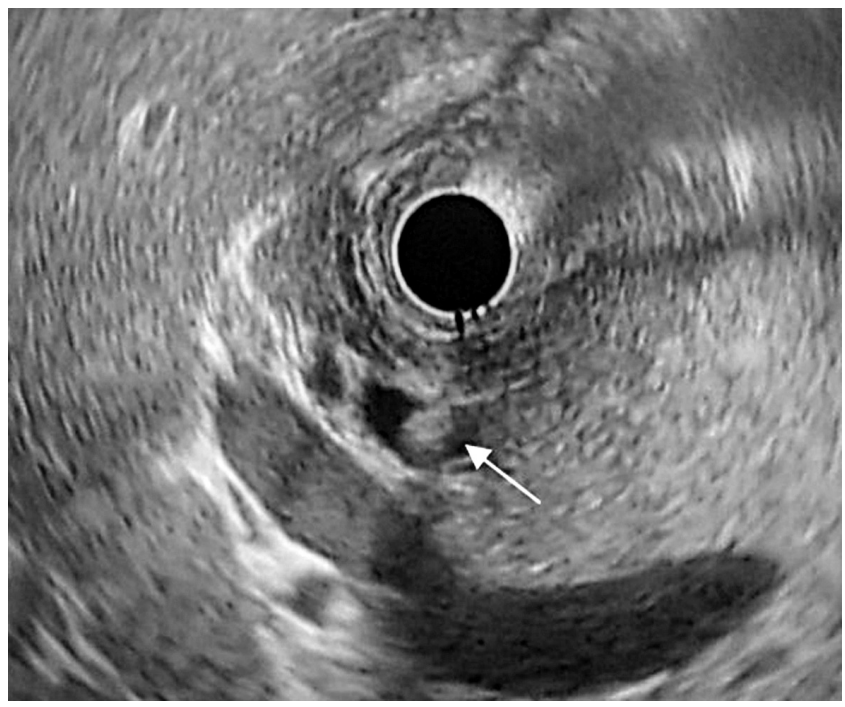

Figura 2. USE previo a CPRE. Colédoco de $6 \mathrm{~mm}$, con defecto hiperecoico en su interior, que proyecta una sombra acústica posterior de aproximadamente $5 \mathrm{~mm} \times 9 \mathrm{~mm}$ de diámetro (flecha) compatible con coledocolitiasis. Imagen propiedad de los autores.

Luego de aplicar los criterios diagnósticos de las guías Tokio de colangitis, se concluye que esta paciente no presenta sospecha diagnóstica ni diagnóstico definitivo de colangitis (Tabla 2).

Tabla 2. Criterios diagnósticos de colangitis

Inflamación sistémica
- A-1 Fiebre $\left(>38^{\circ} \mathrm{C}\right)$ o escalofríos
- A-2 Respuesta inflamatoria en laboratorios (leucocitos $<4000 \mu \mathrm{L} 0$
PCR $>0=1 \mathrm{mg} / \mathrm{dL}$ )
Colestasis
- B-1 Ictericia (bilirrubina total > o = $2 \mathrm{mg} / \mathrm{dL}$ )
- B-2 Pruebas de función hepática alteradas (FA, GGT, AST y ALT).
Elevación más de 1,5 veces el límite superior de la normalidad
Imagen
- C-1 Dilatación de la vía biliar
- C-2 Evidencia de la etiología en imagen (estenosis, cálculo, stent,
etc.)
Sospecha diagnóstica: un ítem A + un ítem B o C
Diagnóstico definitivo: un ítem en A + un ítem en B + un ítem en C.

FA: Fosfatasa alcalina; GGT: gamma-glutamil transferasa. Tomado de: Guías Tokio; 2018.
Se procede a realizar una CPRE; se afronta la papila en eje corto con el papilótomo de arco y la guía hidrofílica, con lo que se observa la salida escasa de bilis por el orificio ampular. Luego se canula la papila dirigiendo la punta del papilótomo al meridiano de las 11 con un avance fácil y profundo de la guía unos $5 \mathrm{~cm}$; posteriormente, se procede a retirar la guía hidrofílica para luego succionar con la jeringa hasta obtener líquido biliar no purulento, lo que confirma que el papilótomo se encuentra en la vía biliar (Figura 3). Se realiza una papilotomía amplia sin complicaciones $y$, posteriormente, se realiza un recambio de papilótomo por un balón extractor. Luego se procede a realizar un barrido, logrando extraer el cálculo; finalmente, se realiza nuevo barrido con la extracción de bilis sin nuevos cálculos. Después de la CPRE se realiza un nuevo USE para corroborar que el colédoco no tiene litiasis en su interior, por lo que no se canula el conducto pancreático.

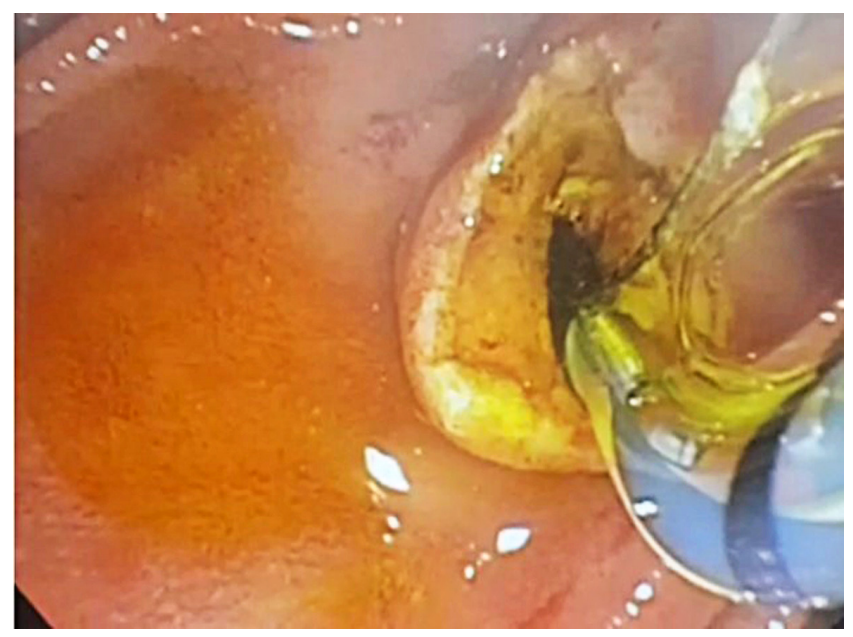

Figura 3. Papilotomía-CPRE. Imagen propiedad de los autores.

La paciente evoluciona satisfactoriamente, lo cual se evidencia por el descenso de bilirrubinas y la disminución progresiva de las transaminasas (Tabla 3). Por parte de cirugía general se programa para realizarle una colecistectomía laparoscópica en la misma hospitalización, procedimiento que no tuvo complicaciones. Después de esto se le dio el egreso.

\section{CASO 2}

Paciente de 37 años con embarazo de 31 semanas, sin antecedentes de importancia, quien presenta un cuadro clínico de aproximadamente 2 meses de evolución caracterizado por episodios de dolor abdominal localizado en el hipocondrio derecho. 4 días antes de la consulta en nuestra institución, el dolor se hace persistente, se asocia con fiebre de $38^{\circ} \mathrm{C}$ y emesis de contenido alimentario, razón por la 
Tabla 3. Paraclínicos después de la CPRE para el caso 1

\begin{tabular}{|lccc|}
\hline \multicolumn{1}{c}{ Examen } & Resultado & Unidades & Valor de referencia \\
\hline TGO-AST & 357 & $\mathrm{U} / \mathrm{L}$ & $0-35$ \\
\hline TGP-ALT & 87 & $\mathrm{U} / \mathrm{L}$ & $0-35$ \\
\hline Bilirrubina total & 1,4 & $\mathrm{mg} / \mathrm{dL}$ & $0,3-1$ \\
\hline Bilirrubina directa & 0,71 & $\mathrm{mg} / \mathrm{dL}$ & $0,0-0,2$ \\
\hline Bilirrubina indirecta & 0,69 & $\mathrm{mg} / \mathrm{dL}$ & $0,0-1,1$ \\
Creatinina & 0,41 & $\mathrm{mg} / \mathrm{dL}$ & $0,5-1,0$
\end{tabular}

cual consulta. En el examen físico de ingreso se documenta: PA: 105/69 mm Hg; FC: 66 lpm; FR: 18 rpm; T: 36,4 ${ }^{\circ} \mathrm{C}$; $\mathrm{SatO}_{2}$ : $94 \%$. Conjuntivas rosadas, escleras con tinte ictérico, mucosa oral húmeda, abdomen blando globoso por útero grávido, dolor a la palpación en el hipocondrio derecho, sin signos de irritación peritoneal y extremidades eutróficas sin edemas. El perfil hepático de ingreso muestra hiperbilirrubinemia a expensas de la bilirrubina directa y patrón colestásico (Tabla 4). Se realiza una ecografía de abdomen total que evidencia una dilatación de la vía biliar en $10 \mathrm{~mm}$ de diámetro en su porción intra- y extrahepática sin identificación de su causa, barro biliar y microlitiasis que ocupa el cuello vesicular, sin signos de colecistitis aguda. Se consideró la realización de una coledocolitiasis de probabilidad intermedia y se realizó colangiorresonacia, que evidenció una dilatación de la vía biliar intra- y extrahepática hasta de $11 \mathrm{~mm}$; en el colédoco intraduodenal se identifica un defecto de $4 \mathrm{~mm}$ de diámetro en su luz. La vesícula biliar tiene paredes delgadas y en su interior se identifica una imagen hipointensa de $3 \mathrm{~mm}$ en el fondo; todos los anteriores hallazgos son compatibles con colelitiasis y coledocolitiasis, sin signos de colecistitis aguda (Figura 4).

Tabla 4. Paraclínicos de ingreso caso 2

\begin{tabular}{|lccc|}
\multicolumn{1}{c}{ Examen } & Resultado & Unidades & Valor de referencia \\
\hline TGO-AST & 36 & $\mathrm{U} / \mathrm{L}$ & $0-35$ \\
\hline TGP-ALT & 31 & $\mathrm{U} / \mathrm{L}$ & $0-35$ \\
\hline Bilirrubina total & 2,29 & $\mathrm{mg} / \mathrm{dL}$ & $0,3-1$ \\
\hline Bilirrubina directa & 1,36 & $\mathrm{mg} / \mathrm{dL}$ & $0,0-0,2$ \\
\hline Bilirrubina indirecta & 0,93 & $\mathrm{mg} / \mathrm{dL}$ & $0,0-1,1$ \\
\hline Creatinina & 0,5 & $\mathrm{mg} / \mathrm{dL}$ & $0,5-1,0$ \\
\hline Fosfatasa alcalina & 121 & $\mathrm{U} / \mathrm{L}$ & $30-120$
\end{tabular}

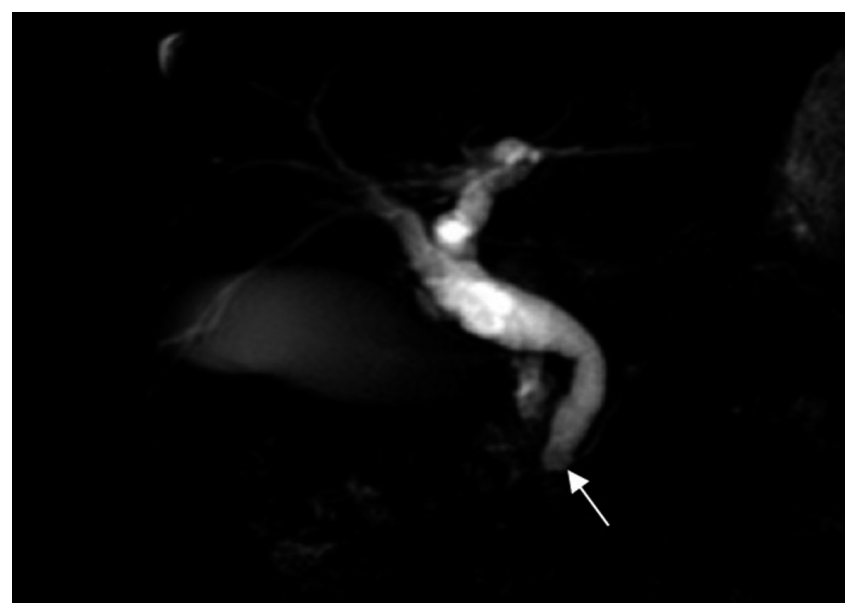

Figura 4. Colangiorresonancia. En el colédoco intraduodenal se observa un defecto de $4 \mathrm{~mm}$ (flecha) en su luz compatible con coledocolitiasis. Imagen propiedad de los autores.

Se decide llevar a la paciente a USE-CPRE. Se realiza un USE en el que se observa una papila en la segunda porción duodenal de aspecto normal y el colédoco dilatado hasta $8 \mathrm{~mm}$ de diámetro con múltiples defectos hiperecoicos en su interior, de aproximadamente $2 \mathrm{~mm}$ a $5 \mathrm{~mm}$, que proyectan una sombra acústica posterior. La vesícula muestra colelitiasis sin colecistitis, y el páncreas tiene un aspecto endosonográfico normal. Se retira el USE radial y se inserta un duodenoscopio (Figura 5).

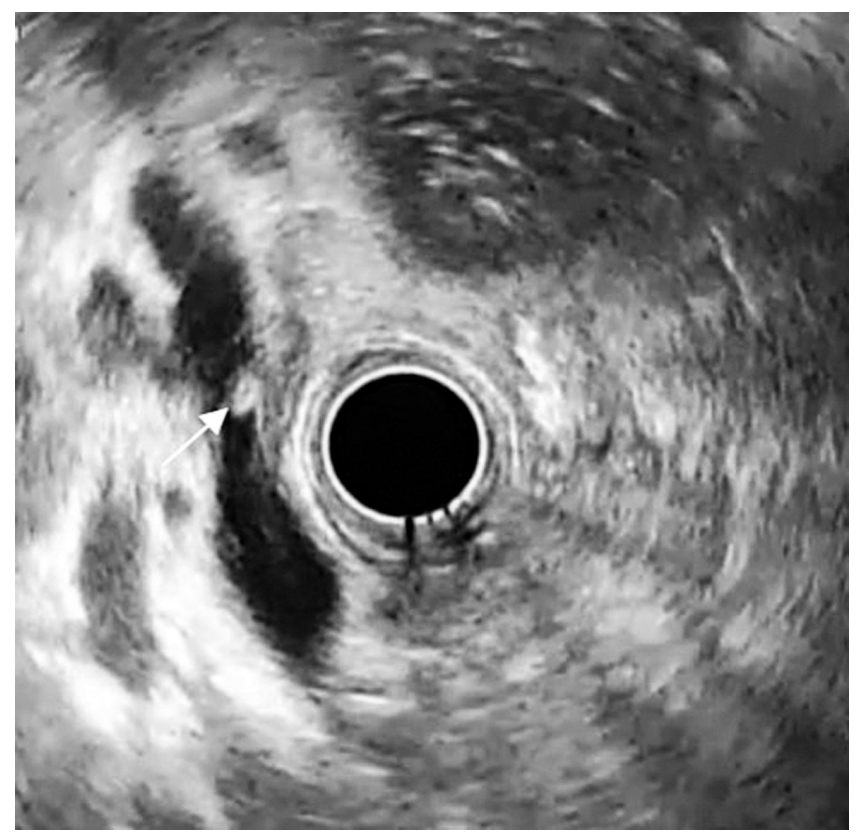

Figura 5. USE previo a la CPRE. Colédoco dilatado hasta $8 \mathrm{~mm}$ de diámetro, con defecto hiperecoico en su interior de aproximadamente $5 \mathrm{~mm}$ (flecha), que proyecta una sombra acústica posterior compatible con coledocolitiasis. Imagen propiedad de los autores. 
Se procede a realizar una CPRE usando la misma técnica descrita con la paciente anterior (Figura 6). No se usa fluoroscopio para proteger al feto de la radiación.

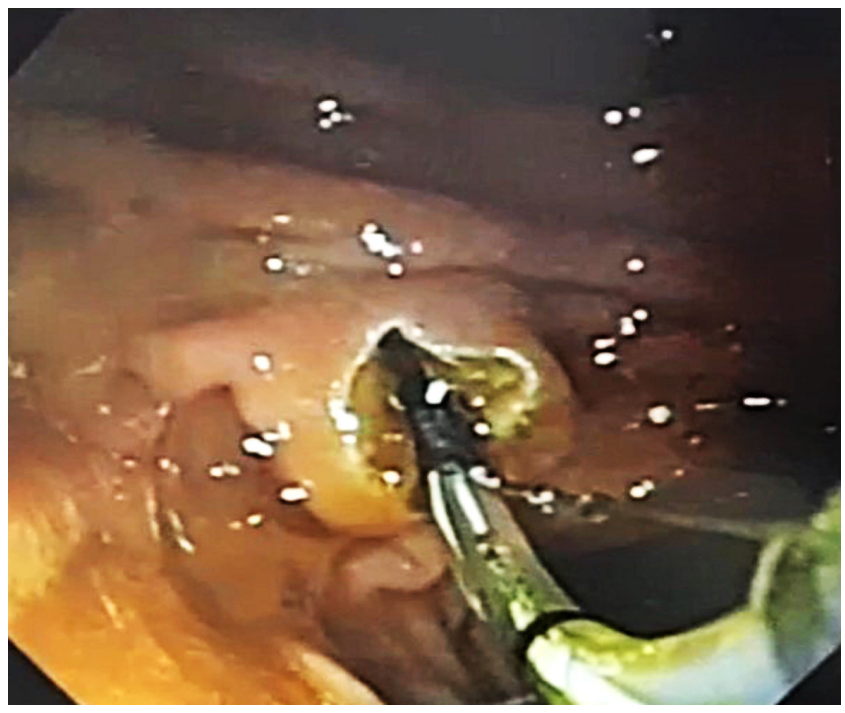

Figura 6. Papilotomía-CPRE. Imagen propiedad de los autores.

El procedimiento se realizó sin complicaciones. La paciente evolucionó favorablemente en el posoperatorio, con una mejoría clínica y paraclínica dada por la disminución de las bilirrubinas y el descenso progresivo de las transaminasas (Tabla 5). Se programa para la realización de una colecistectomía laparoscópica de manera ambulatoria.

Tabla 5. Paraclínicos después de la CPRE para el caso 2

\section{Examen Resultado Unidades Valor de referencia}

$\begin{array}{lccc}\text { TGO-AST } & 38 & \mathrm{U} / \mathrm{L} & 0-35 \\ \text { TGP-ALT } & 40 & \mathrm{U} / \mathrm{L} & 0-35 \\ \text { Bilirrubina total } & 0,84 & \mathrm{mg} / \mathrm{dL} & 0,3-1 \\ \text { Bilirrubina directa } & 0,32 & \mathrm{mg} / \mathrm{dL} & 0,0-0,2 \\ \text { Bilirrubina indirecta } & 0,52 & \mathrm{mg} / \mathrm{dL} & 0,0-1,1 \\ \text { Fosfatasa alcalina } & 98 & \mathrm{U} / \mathrm{L} & 30-120\end{array}$

Se aplican los criterios de Tokio para colangitis; en esta paciente se cumplen los criterios B y C, por lo que no se establece una sospecha diagnóstica ni definitiva de colangitis (Tabla 2) ${ }^{(7)}$.

\section{DISCUSIÓN}

La enfermedad litiásica biliar es una condición frecuente en mujeres embarazadas, favorecida por la disminución en la motilidad de la vesícula. Este hecho da lugar a un menor vaciamiento asociado con la elevación en los niveles de colesterol saturado en la bilis, lo cual contribuye al aumento de la litogenicidad. Todos estos fenómenos son desencadenados por la progesterona y los estrógenos ${ }^{(8)}$.

Entre las complicaciones de la enfermedad litiásica biliar en las pacientes gestantes está la coledocolitiasis, condición poco frecuente (incidencia de 1/1000 embarazos), pero que de no ser manejada adecuadamente puede llevar a complicaciones y morbilidad tanto materna como fetal ${ }^{(3,9)}$.

En los últimos años se han venido implementando técnicas de CPRE sin fluoroscopia con el objetivo de prevenir efectos estocásticos en el feto. Akcakaya y colaboradores en 2009 describieron una serie de casos de 6 pacientes embarazadas intervenidas mediante CPRE sin fluoroscopia, en la que reportaron que ninguna de ellas presentó complicaciones pos-CPRE, abortos, partos pretérmino ni malformaciones en el feto ${ }^{(10)}$.

En 2016 Ersoz y colaboradores publicaron una serie de casos retrospectivos de pacientes embarazadas intervenidas con CPRE sin radiación, que incluyó 22 pacientes a quienes se les realizó una esfinterotomía biliar y dilatación neumática con balón de la papila; de esta serie, 2 pacientes presentaron pancreatitis pos-CPRE y una hemorragia digestiva asociada con la esfinterotomía. En todos los casos el embarazo fue normal y finalizó satisfactoriamente con el nacimiento de un niño sano ${ }^{(11)}$. Sin embargo, es importante destacar que cuando el USE se asocia con la CPRE alcanza un éxito terapéutico mayor; esto lo demostraron Huang y colaboradores, quienes en 2013 compararon 32 mujeres embarazadas, a quienes se les realizó una CPRE guiada por USE frente a 36 mujeres embarazadas, a quienes se les realizó una CPRE sin ultrasonido, y encontraron una tasa de éxito del $90 \%$ en las primeras en comparación con el $60 \%$ en el otro grupo ${ }^{(12)}$.

Estudios recientes aportan nueva evidencia sobre la seguridad de la CPRE convencional con fluoroscopia en el embarazo, como lo demostraron Azad y colaboradores; en 2019 realizaron una revisión sistemática y un metaanálisis que incluyó 1307 pacientes embarazadas intervenidas con CPRE con fluoroscopia y sin fluoroscopia, en el que no se encontraron diferencias en términos de complicaciones maternas ni fetales ${ }^{(13)}$.

Los efectos estocásticos en el feto, atribuidos a la exposición a la radiación, posiblemente no son evidentes en los diferentes estudios debido a que no tienen un un tiempo de seguimiento suficiente; sin embargo, el principio básico de seguridad radiológica ALARA (As Low As Reasonably Achievable), que significa tan bajo como sea razonablemente alcanzable, considera que no hay un nivel de radiación seguro, y que siempre se debe dar la dosis de radiación tan baja como sea posible para prevenir efectos estocásticos a futuro ${ }^{(14)}$. 
Cabe destacar que la no exposición a la radiación en las pacientes gestantes disminuye la presentación de efectos estocásticos en el feto ${ }^{(14)}$. Por tal razón, la técnica combinada USE-CPRE se considera de elección para el abordaje de las pacientes embarazadas con coledocolitiasis, pues no expone al feto a la radiación, evento potencialmente generador de complicaciones a futuro; por otro lado, la realización permite una adecuada objetivización de la presencia de cálculos en el colédoco, así como la verificación de la resolución posterior a la CPRE y un mayor éxito terapéutico comparado con la CPRE sin USE ${ }^{(12,15)}$. En algunas series incluso no se consideró realizar la CPRE después de un resultado negativo para coledocolitiasis luego de la USE ${ }^{(15-17)}$.

En la actualidad la CPRE es una técnica esencialmente terapéutica y está indicada en pacientes embarazadas como tratamiento en coledocolitiasis sintomática, colangitis y pancreatitis biliar asociada con obstrucción de la vía biliar persistente. Entre los riesgos habituales asociados con la CPRE están la perforación duodenal en el 0,08 \%-0,6 \%, colangitis en el $0,5 \%$ a $3 \%$, colecistitis aguda en el 0,5\%, sangrado con una tasa de $0,3 \%$ a $2 \%$ y pancreatitis en $12 \%$; estas complicaciones pueden traer consecuencias para la madre y el feto, además se ha documentado en diferentes estudios que se presentan con la misma frecuencia en mujeres embarazadas y no embarazadas.

La CPRE no debe ser aplazada en pacientes con indicación clara, pues posponer el procedimiento se asocia con complicaciones maternas y fetales subsiguientes ${ }^{(3,18,19)}$. La exposición a la radiación durante la fluoroscopia mientras se realiza la CPRE y sus potenciales efectos adversos al producto de la gestación forman parte de los mayores temores asociados con el procedimiento tanto para los médicos como para las pacientes; los efectos estocásticos que se presentan independientemente de la dosis de radiación recibida son los que más preocupan, como es el caso de las alteraciones genéticas en el feto y potencial carcinogénesis (leucemias, cáncer infantil, linfomas, tumores del sistema nervioso central), que se presentan incluso con dosis menores de $100 \mathrm{mGy}$ y son independientes de la dosis ${ }^{(14)}$. Los efectos determinísticos, como el retraso en el crecimiento intrauterino y alteraciones en el desarrollo cognitivo, requieren de dosis mayores de $100 \mathrm{mGy}$, umbral muy por encima de las dosis recibidas cuando se realiza una CPRE con fluoroscopia por un endoscopista experto, la cual llega a ser aproximadamente de $0,1 \mathrm{a} 3 \mathrm{mGy}^{(3)}$.

Teniendo en cuenta los efectos estocásticos de la radiación asociada con la CPRE, en los últimos años se han venido implementando intervenciones para minimizar la exposición a la radiación de mujeres embarazadas durante la CPRE, entre las que se describen: el uso de un mandil plomado que cubra la pelvis materna, utilizar el menor tiempo posible la fluoroscopia y la realización de CPRE sin fluoroscopia. Varios estudios han demostrado que la implementación de esta técnica es un procedimiento seguro y efectivo en el tratamiento de la coledocolitiasis durante el embarazo $^{(3,11,20)}$.

El USE, como herramienta adicional realizada al mismo tiempo de la CPRE, permite complementar efectivamente el procedimiento de extracción de cálculos dado que establece el número y la localización de cálculos antes de la CPRE y luego de la CPRE más extracción; la USE permite evaluar el conducto biliar común y de esta forma corroborar que el colédoco presenta una adecuada permeabilidad. Por otro lado, en algunas series se ha documentado que luego de realizar el USE, si no se observan cálculos, se evita la realización de la $\operatorname{CPRE}^{(16,17)}$.

\section{CONCLUSIÓN}

El estudio y tratamiento de la mujer embarazada con coledocolitiasis requiere de la aproximación clínica detallada, la indicación e interpretación adecuadas de estudios de laboratorio e imagen y la realización de técnicas de intervención de la vía biliar que minimicen la exposición fetal a la radiación ionizante, y permitan de una manera confiable confirmar la resolución de la coledocolitiasis; estos objetivos se cumplen a cabalidad con la técnica combinada de USE-CPRE. Por esta razón, en nuestra institución se cuenta con la tecnología y el personal entrenado para realizar este tipo de intervenciones, lo cual minimiza los riesgos para la madre y para el feto. Se describen dos casos de gestantes con coledocolitiasis, a quienes se les realizó USE-CPRE, intervención con la que logramos no exponer al producto de la gestación a la radiación ionizante y pudimos resolver exitosamente la coledocolitiasis sin complicaciones para el binomio madre e hijo.

\section{Financiación}

Los autores declaran no haber recibido financiación alguna para la realización del trabajo.

\section{Conflictos de interés}

Los autores declaran no tener ningún conflicto de interés.

\section{Agradecimientos}

A todos los integrantes de la Unidad de Gastroenterología y Endoscopia Digestiva del Hospital Universitario San Ignacio, en la ciudad de Bogotá, Colombia. 
1. Everson G. Pregnancy and Gallstones. Hepatology. 1993; 17(1):159-61.

https://doi.org/10.1002/hep.1840170126

2. Ibiebele I, Schnitzler M, Nippita T, Ford JB. Outcomes of Gallstone Disease during Pregnancy: a Population-based Data Linkage Study. Paediatric and Perinatal Epidemiology. 2017;31(6):522-30. https://doi.org/10.1111/ppe.12406

3. Arce-Liévano E, del Río-Suárez I, Valenzuela-Salazar C, Délano-Alonso R, Bada-Yllán O, Herrera-Esquivel JJ, Moreno-Portillo M. Endoscopic retrograde cholangiopancreatography results for the treatment of symptomatic choledocholithiasis in pregnant patients: A recent experience at a secondary care hospital in Mexico City. Revista de Gastroenterología de México. 2021;86(1): 21-27. https://doi.org/10.1016/j.rgmxen.2019.12.002

4. Chan $\mathrm{CH}$, Enns RA. ERCP in the management of choledocholithiasis in pregnancy. Curr Gastroenterol Rep. 2012;14(6):504-10. https://doi.org/10.1007/s11894-012-0294-0

5. Konduk BT, Bayraktar O. Efficacy and safety of endoscopic retrograde cholangiopancreatography in pregnancy: A high-volume study with long-term follow-up. Turkish Journal of Gastroenterology. 2019;30(9):811-6. https://doi.org/10.5152/tjg.2019.18799

6. Riley DS, Barber MS, Kienle GS, Aronson JK, von SchoenAngerer T, Tugwell P, Kiene H, Helfand M, Altman DG, Sox H, Werthmann PG, Moher D, Rison RA, Shamseer L, Koch CA, Sun GH, Hanaway P, Sudak NL, Kaszkin-Bettag $\mathrm{M}$, Carpenter JE, Gagnier JJ. CARE guidelines for case reports: explanation and elaboration document. Journal of Clinical Epidemiology. 2017;89:218-35. https://doi.org/10.1016/j.jclinepi.2017.04.026

7. Kiriyama S, Kozaka K, Takada T, Strasberg SM, Pitt HA, Gabata T, Hata J, Liau KH, Miura F, Horiguchi A, Liu KH, Su CH, Wada K, Jagannath P, Itoi T, Gouma DJ, Mori Y, Mukai S, Giménez ME, Huang WS, Kim MH, Okamoto K, Belli G, Dervenis C, Chan ACW, Lau WY, Endo I, Gomi H, Yoshida M, Mayumi T, Baron TH, de Santibañes E, Teoh AYB, Hwang TL, Ker CG, Chen MF, Han HS, Yoon YS, Choi IS, Yoon DS, Higuchi R, Kitano S, Inomata M, Deziel DJ, Jonas E, Hirata K, Sumiyama Y, Inui K, Yamamoto M. Tokyo Guidelines 2018: diagnostic criteria and severity grading of acute cholangitis (with videos). Journal of HepatoBiliary-Pancreatic Sciences. 2018;25(1):17-30. https://doi.org/10.1002/jhbp.512

8. González-Zúñiga AM. Choledocholithiasis in pregnancy. Case report. Clinica e Investigacion en Ginecologia y Obstetricia. 2019;46(3):122-6. https://doi.org/10.1016/j.gine.2019.01.001

9. Sethi S, Thosani N, Banerjee S. Radiation-free ERCP in pregnancy: A "sound" approach to leaving no stone untur- ned. Dig Dis Sci. 2015;60(9):2604-7. https://doi.org/10.1007/s10620-014-3502-y

10. Akcakaya A, Ozkan OV, Okan I, Kocaman O, Sahin M. Endoscopic retrograde cholangiopancreatography during pregnancy without radiation. World Journal of Gastroenterology. 2009; 15(29):3649-52. https://doi.org/10.3748/wjg.15.3649

11. Ersoz G, Turan I, Tekin F, Ozutemiz O, Tekesin O. Nonradiation ERCP with endoscopic biliary sphincterotomy plus papillary balloon dilation for the treatment of choledocholithiasis during pregnancy. Surgical Endoscopy. 2016;30(1):222-8. https://doi.org/10.1007/s00464-015-4190-1

12. Huang $\mathrm{P}$, Zhang H, Zhang XF, Zhang X, Lü W, Fan Z. Comparison of endoscopic retrograde cholangiopancreatography performed without radiography and with ultrasound-guidance in the management of acute pancreaticobiliary disease in pregnant patients. Chinese Medical Journal. 2013;126(1):46-50.

13. Azab M, Bharadwaj S, Jayaraj M, Hong AS, Solaimani P, Mubder M, Yeom H, Yoo JW, Volk ML. Safety of endoscopic retrograde cholangiopancreatography (ERCP) in pregnancy: A systematic review and meta-analysis. Saudi J Gastroenterol. 2019;25(6):341-354. https://doi.org/10.4103/sjg.SJG_92_19

14. Vock P. Clinical perspective on diagnostic X-ray examinations of pregnant patients - What to take into account. Physica Medica. 2017;43:165-71. https://doi.org/10.1016/j.ejmp.2017.05.004

15. Girotra M, Jani N. Role of endoscopic ultrasound/ SpyScope in diagnosis and treatment of choledocholithiasis in pregnancy. World J Gastroenterol. 2010;16(28):3601-2. https://doi.org/10.3748/wjg.v16.i28.3601

16. Vohra S, Holt EW, Bhat YM, Kane S, Shah JN, Binmoeller KF. Successful single-session endosonography-based endoscopic retrograde cholangiopancreatography without fluoroscopy in pregnant patients with suspected choledocholithiasis: A case series. Journal of Hepato-BiliaryPancreatic Sciences. 2014;21(2):93-7. https://doi.org/10.1002/jhbp.7

17. Shah JN, Bhat YM, Hamerski CM, Kane SD, Binmoeller KF. Feasibility of nonradiation EUS-based ERCP in patients with uncomplicated choledocholithiasis (with video). Gastrointestinal Endoscopy. 2016:84(5):764-9. https://doi.org/10.1016/j.gie.2016.03.1485

18. Magno-Pereira V, Moutinho-Ribeiro P, Macedo G. Demystifying endoscopic retrograde cholangiopancreatography (ERCP) during pregnancy. Eur J Obstet Gynecol Reprod Biol. 2017;219:35-39. https://doi.org/10.1016/j.ejogrb.2017.10.008

19. Inamdar S, Berzin TM, Sejpal DV, Pleskow DK, Chuttani $\mathrm{R}$, Sawhney MS, Trindade AJ. Pregnancy is a Risk 
Factor for Pancreatitis After Endoscopic Retrograde

Cholangiopancreatography in a National Cohort Study.

Clin Gastroenterol Hepatol. 2016;14(1):107-14.

https://doi.org/10.1016/j.cgh.2015.04.175
20. Agcaoglu O, Ozcinar B, Gok AF, Yanar F, Yanar H, Ertekin $\mathrm{C}$, Gunay K. ERCP without radiation during pregnancy in the minimal invasive world. Arch Gynecol Obstet. 2013;288(6):1275-8.

https://doi.org/10.1007/s00404-013-2890-0 